\title{
Bibliography
}

1. P. A. White, Some characterizations of generalized manifolds with boundaries, not yet published.

2. R. L. Wilder, Topology of manifolds, Amer. Math. Soc. Colloquium Publications, vol. 32, 1949.

University of SoUthern California

\section{THE BEHAVIOR AT ZERO OF THE CHARACTERISTIC FUNCTION OF A RANDOM VARIABLE}

\section{ROSENBLATT ${ }^{1}$}

The weak laws of probability theory dealing with the asymptotic behavior of normed sums of independent and identically distributed random variables can of ten be rephrased to relate the behavior at zero of the characteristic function of a random variable to the behavior of the distribution function of the random variable at infinity. A few simple theorems of this type are here obtained.

Let $X$ be a random variable with distribution function $F(x)$ and characteristic function $\phi(z)=\int_{-\infty}^{\infty} e^{i z x} d F(x)$. Let $a(u)$ be a strictly increasing positive function defined for $u>0$ that diverges to $\infty$ as $u \rightarrow \infty . a^{-1}(u)$ will denote the inverse function of $a(u)$. The following theorems will be proved:

THEOREM 1. If

$$
\frac{a(u+1)}{a(u)}<K \quad \text { for } u \geqq 1
$$

and

$$
\frac{n}{a(n)^{2}} \int_{1}^{a(n)} \frac{x d x}{a^{-1}(x)} \leqq K, \quad \frac{n}{a(n)^{2}}=o(1) \quad \text { as } n \rightarrow \infty
$$

for some constant $K$, then

$$
1-\phi(z)=o\left(\frac{1}{a^{-1}(1 / z)}\right) \quad \text { at } z=0
$$

if and only if

Received by the editors March 2, 1951 and, in revised form, November 10, 1951.

1 This work was supported by the Office of Naval Research. 


$$
\begin{aligned}
n \int_{|x|>a(n)} d F(x) & =o(1), \\
\frac{1}{a(n)} \sum_{k=1}^{n} \int_{|x|<a(k)} x d F(x) & =o(1) .
\end{aligned}
$$

THEOREM 2. $1-\phi(z)=o\left(|z|^{\alpha}\right)$ at $z=0$ if and only if

(i) $n \int_{|x|>n^{1 / \alpha}} d F(x)=o$ (1) as $n \rightarrow \infty$, if $0<\alpha<1$.

(ii) $n \int_{|x|>n} d F(x)=o(1), \int_{|x|<n} x d F(x)=o(1)$ as $n \rightarrow \infty$, if $\alpha=1$.

(iii) $n \int_{|x|>n^{1 / \alpha}} d F(x)=o(1)$ as $n \rightarrow \infty$ and the mean $\int_{-\infty}^{\infty} x d F(x)=0$, if $1<\alpha<2$.

Theorem 2 is a corollary of Theorem 1 obtained by setting $a(u)$ $=u^{1 / \alpha}$. The preceding theorems could be proved directly. However, the purpose of the paper is to derive the theorems by rephrasing a special case of the weak law of large numbers.

The following is a statement of W. Feller's [2] necessary and sufficient conditions for the weak law of large numbers for independent and identically distributed random variables:

Let $X_{k}(k=1,2, \cdots)$ be independent and identically distributed random variables with distribution function $F(x)$. Let $s$ be a median of $X_{k}$, i.e., let $F(s+) \geqq \lambda>0, F(s-) \leqq 1-\lambda$. A necessary and sufficient condition that there be a sequence $b_{k}$ such that

$$
\frac{1}{a(n)} \sum_{k=1}^{n}\left(X_{k}-b_{k}\right) \rightarrow 0
$$

in probability

is that

$$
\begin{aligned}
n \int_{|x|>a(n)} d F(x+s) & =o(1), \\
\frac{n}{a(n)^{2}} \int_{|x|<a(n)} x^{2} d F(x+s) & =o(1) .
\end{aligned}
$$

It suffices to take $b_{k}=\int_{|x|<a(k)} x d F(x)$.

Feller's conditions are needed in a slightly different form. Conditions (3), (4) can be replaced by the following conditions

$$
\begin{aligned}
n \int_{|x|>a(n)} d F(x) & =o(1), \\
\frac{n}{a(n)^{2}} \int_{|x|<a(n)} x^{2} d F(x) & =o(1)
\end{aligned}
$$

if the $X_{k}$ are not constants. Conditions (3) and (4) immediately imply 
condition (5). Condition (4) implies that $n / a(n)^{2} \rightarrow 0$ since the $X_{k}$ are not constants. But

$$
\begin{aligned}
0 \leqq & \frac{n}{a(n)^{2}} \int_{|x|<a(n)} x^{2} d F(x)=\frac{n}{a(n)^{2}} \int_{-a(n)-s}^{a(n)-s}\left(x^{2}+2 x s+s^{2}\right) d F(x+s) \\
\leqq & \frac{n}{a(n)^{2}} \int_{|x|<a(n)+|s|} x^{2} d F(x+s) \\
& +\frac{2|s| n}{a(n)^{2}}\left|\int_{|x|<a(n)+|s|} x^{2} d F(x+s)\right|^{1 / 2} \\
& +\frac{s^{2} n}{a(n)^{2}} \int_{-\infty}^{\infty} d F(x+s)
\end{aligned}
$$

where the second term of the second line of the inequality is obtained by the Schwarz inequality. Further

$$
\begin{aligned}
\frac{n}{a(n)^{2}} \int_{|x|<a(n)+|s|} x^{2} d F(x+s) & \leqq \frac{n}{a(n)^{2}} \int_{|x|<a(n)} x^{2} d F(x+s) \\
+ & \frac{[a(n)+|s|]^{2}}{a(n)^{2}} n \int_{|x|<a(n)+|s|} d F(x+s)=o(1)
\end{aligned}
$$

and

$$
\frac{s^{2} n}{a(n)^{2}} \int_{-\infty}^{\infty} d F(x+s)=\frac{s^{2} n}{a(n)^{2}}=o(1)
$$

Therefore

$$
\frac{n}{a(n)^{2}} \int_{|x|<a(n)} x^{2} d F(x)=o(1) .
$$

Similar reasoning can be used to show that (5), (6) imply (3), (4). Hence the two sets of conditions are equivalent. ${ }^{2}$

Lemma 1. Assume condition (2). Then (5) is a necessary and suffcient condition that there be a sequence $b_{k}$ such that

$$
\frac{1}{a(n)} \sum_{k=1}^{n}\left(X_{k}-b_{k}\right) \rightarrow 0 \quad \text { in probability. }
$$

Given $x$ sufficiently large, there is an $n$ such that $n \leqq a^{-1}(x)<n+1$. The inequality

2 The author would like to thank the referee for suggesting the simple proof given above of the equivalence of conditions (3), (4) and (5), (6). 


$$
n \int_{|y|>a(n+1)} d F(y) \leqq a^{-1}(x) \int_{|y|>x} d F(y) \leqq(n+1) \int_{|y|>a(n)} d F(y)
$$

and condition (5) imply that

$$
a^{-1}(x) \int_{|y|>x} d F(y)=o(1)
$$

as $x \rightarrow \infty$. Let

$$
V(x)=1-F(x)+F(-x)
$$

Now

$$
\begin{aligned}
\frac{n}{a(n)^{2}} \int_{|x|<a(n)} x^{2} d F(x) & =\frac{-n}{a(n)^{2}} \int_{0}^{a(n)} x^{2} d V(x) \\
= & -n V(a(n))+\frac{n}{a(n)^{2}}\left(\int_{0}^{1}+\int_{1}^{a(n)}\right) 2 x V(x) d x .
\end{aligned}
$$

The first two terms on the right-hand side of the preceding equality are $o(1)$ and

$$
\begin{aligned}
\frac{n}{a(n)^{2}} \int_{1}^{a(n)} 2 x V(x) d x & =\frac{2 n}{a(n)^{2}} \int_{1}^{a(n)} \frac{x}{a^{-1}(x)} a^{-1}(x) V(x) d x \\
& =o\left(\frac{n}{a(n)^{2}} \int_{1}^{a(n)} \frac{x}{a^{-1}(x)} d x\right)=o(1),
\end{aligned}
$$

by $\left(5^{\prime}\right)$. Hence

$$
\frac{n}{a(n)^{2}} \int_{|x|<a(n)} x^{2} d F(x)=o(1)
$$

Lemma 2. Assume condition (1). Then $n \log \phi(x / a(n)) \rightarrow 0$ for all $x, n \rightarrow \infty$, is equivalent to $1-\phi(z)=o\left(1 / a^{-1}(1 / z)\right)$ at $z=0$.

Let $n \log \phi(x / a(n)) \rightarrow 0$ for all $x$. Then $n \log \phi(x / a(n)) \rightarrow 0$ uniformly in every finite interval as $n \rightarrow \infty$ [3]. Given $z$ sufficiently small, condition (1) implies that $x$ and $n$ can be obtained such that $x / a(n)$ $=z$ and $1 \leqq|x| \leqq 2 K$. Then

$$
\begin{aligned}
\left|a^{-1}\left(\frac{1}{z}\right) \log \phi(z)\right| & =\left|\frac{a^{-1}(1 / z)}{a^{-1}(x / z)} a^{-1}(x / z) \log \phi(z)\right| \\
& \leqq|n \log \phi(x / a(n))| \rightarrow 0 .
\end{aligned}
$$

But $-[\log \phi(z)] /(1-\phi(z)) \rightarrow 1$ as $z \rightarrow 0$. Hence $a^{-1}(1 / z)(1-\phi(z))$ $=o(1)$ at $z=0$. 
Let $a^{-1}(1 / z)(1-\phi(z))=o(1)$ at $z=0$. Given $|x|<1, n$ and $z$ can be obtained such that $x / a(n)=z$. Then

$$
\begin{aligned}
|n \log \phi(x / a(n))| & =\left|\frac{a^{-1}(x / z)}{a^{-1}(1 / z)} a^{-1}(1 / z) \log \phi(z)\right| \\
& \leqq\left|a^{-1}(1 / z) \log \phi(z)\right| \rightarrow 0
\end{aligned}
$$

and we must have

$$
n \log \phi(x / a(n)) \rightarrow 0
$$

for $|x|<1$. This implies that $n \log \phi(x / a(n)) \rightarrow 0$ for all $x$. For if $\phi(x / a(n))^{n}$ has a limit as $n \rightarrow \infty$ for all $x$, this limit must be 1 by the continuity theorem for characteristic functions [1] since 1 is the only characteristic function equal to 1 in a neighborhood of the origin. But $\phi(x / a(n))^{n}$ must have a limit for all $x$ as $n \rightarrow \infty$. If this were not so, one could extract two convergent subsequences having a different limiting value for some $x,|x|>1$. But the continuity theorem implies that both sequences have the limit 1 leading us to a contradiction.

Now

$$
n \log \phi(x / a(n)) \rightarrow 0
$$

for all $x$ as $n \rightarrow \infty$ is equivalent to

$$
\frac{1}{a(n)} \sum_{k=1}^{n} X_{k} \rightarrow 0
$$

in probability [1]. This remark indicates that Theorem 1 follows immediately from Lemmas 1 and 2 .

The function $a(u)=u^{1 / \alpha}$ satisfies conditions (1), (2) of Theorem 1. Hence

$$
1-\phi(z)=o\left(|z|^{\alpha}\right)
$$

at $z=0$ if and only if

$$
\begin{aligned}
n \int_{|x|>n^{1 / \alpha}} d F(x) & =o(1), \\
\frac{1}{n^{1 / \alpha}} \sum_{k=1}^{n} \int_{|x|<k^{1 / \alpha}} x d F(x) & =o(1) .
\end{aligned}
$$

Proof of Theorem 2. Let $0<\alpha<1$. Then condition (8) follows from condition (7). 


$$
\begin{aligned}
& \frac{1}{n^{1 / \alpha}} \sum_{k=1}^{n} \int_{|x|<k^{1 / \alpha}} x d F(x) \\
& =\frac{1}{n^{1 / \alpha}} \sum_{k=1}^{n} \sum_{m=1}^{n} \int_{(m-1)^{1 / \alpha} \leqq|x|<m^{1 / \alpha}} x d F(x) \\
& =\frac{1}{n^{1 / \alpha}} \sum_{m=1}^{n}(n-m+1) \int_{(m-1)^{1 / \alpha} \leqq|x| \leqq m^{1 / \alpha}} x d F(x) \\
& =\frac{n}{n^{1 / \alpha}} \int_{|x|<n^{1 / \alpha}} x d F(x)-\sum_{m=1}^{n} \frac{1}{n^{1 / \alpha}} \int_{(m-1)^{1 / \alpha}|x|<m^{1 / \alpha}}(m-1) x d F(x) .
\end{aligned}
$$

Both of these two terms are $o(1)$. The last term is dominated by

$$
\begin{aligned}
\frac{1}{n^{1 / \alpha}} \int_{|x|<n^{1 / \alpha}}|x|^{1+\alpha} d F(x) & =\frac{-1}{n^{1 / \alpha}} \int_{0}^{n^{1 / \alpha}} x^{1+\alpha} d V(x) \\
& =-n V\left(n^{1 / \alpha}\right)+\frac{(1+\alpha)}{n^{1 / \alpha}} \int_{0}^{n^{1 / \alpha}} x^{a} V(x) d x \\
& =o(1)+\frac{(1+\alpha)}{n^{1 / \alpha}} \int_{0}^{n^{1 / \alpha}} o(1) d x=o(1) .
\end{aligned}
$$

The first term

$$
n^{1-1 / \alpha} \int_{|x|<n^{1 / \alpha}} x d F(x)=-n V\left(n^{1 / \alpha}\right)+n^{1-1 / \alpha} \int_{0}^{n^{1 / \alpha}} V(x) d x .
$$

Let $K(\epsilon)$ be such that $|V(x)|<\epsilon / x^{a}$ for $x>K(\epsilon), \epsilon>0$. Then

$$
\left|n^{1-1 / \alpha} \int_{0}^{n^{1 / \alpha}} V(x) d x\right| \leqq n^{1-1 / \alpha} K+\frac{\epsilon n^{1-1 / \alpha}}{1-\alpha}\left(n^{1 / \alpha-1}-K^{1 / \alpha-1}\right)
$$

so that

$$
n^{1-1 / \alpha} \int_{|x|<n^{1 / \alpha}} x d F(x)=o(1)
$$

ii and iii. The argument used in the proof of $i$ to show that

$$
n^{1-1 / \alpha} \int_{|x|<n^{1 / \alpha}} x d F(x)=o(1)
$$

still holds. This reduces to

$$
\int_{|x|<n} x d F(x)=o(1)
$$


when $\alpha=1$. When $\alpha>1$, the condition can be simplified. Then

$$
\int_{-\infty}^{\infty}|x| d F(x)=-\int_{0}^{\infty} x d V(x)=\int_{0}^{\infty} V(x) d x
$$

and the last integral is finite since

$$
|V(x)|=o\left(1 / x^{\alpha}\right)
$$

as $x \rightarrow \infty$. The first moment exists and is zero. On the other hand, the existence of a vanishing first moment implies that

$$
n^{1-1 / \alpha} \int_{|x|<n^{1 / \alpha}} x d F(x)=o(1) .
$$

Now

$$
\begin{aligned}
n^{1-1 / \alpha} \int_{|x|<n^{1 / \alpha}} x d F(x) & =-n^{1-1 / \alpha} \int_{0}^{n^{1 / \alpha}} x d V(x) \\
& =-n V\left(n^{1 / \alpha}\right)+n^{1-1 / \alpha} \int_{0}^{n^{1 / \alpha}} V(x) d x=o(1)
\end{aligned}
$$

since $|V(x)|=o\left(1 / x^{\alpha}\right)$. The proof of part iii is complete.

\section{REFERENCES}

1. H. Cramer, Mathematical methods of statistics, Princeton, 1946, pp. 96-98.

2. W. Feller, Über das Gesetz der grossen Zahlen, Acta Univ. Szeged. vol. 8 (19361937) pp. 191-201.

3. P. Lévy, Theorie de l'addition des variables aléatoires, Paris, 1937, pp. 48-49.

University of Chicago 